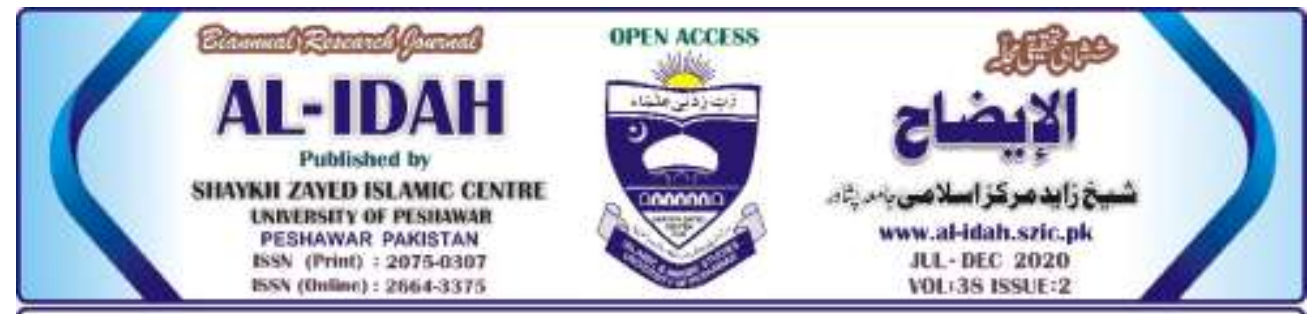

$$
\text { القائد الأعظم محمد علي جناح في الأدب العربي }
$$

\title{
The Quaid Azam Muhammad Ali Jinnah in Arabic Literature
}

\section{Author(s): 1. Uzma Elahi}

PhD Scholar, Dept: of Arabic, UoP, KpK,Email: druzmaelahi@gmail.com

\section{Yaqoob Khan Marwat}

Professor, Dept: of Arabic, UoP, KpK, Email: yaqoobkhan@uop.edu.pk
Issue: http://al-idah.szic.pk/index.php/al-idah/index

URL: http://al-idah.szic.pk/index.php/al-idah/article/view/516

Citation: Elahi, U. and Yaqoob Khan Marwat 2020. The Quaid Azam Muhammad Ali Jinnah in Arabic Literature. Al-Idah . 38, - 2 (Dec. 2020), 67 - 81

Publisher: Shaykh Zayed Islamic Centre, University of Peshawar, Al-Idah - Vol: 38 Issue: 2 / July - Dec 2020/ P.67-81
Article DOI:

https://doi.org/10.37556/a

l-idah.038.02.516

Received on: 13-08-2020

Accepted on:14-10-2020

Published on: 25-122020

The Arab traders introduced the people of the subcontinent to Islam and Muslim civilization. The scholars of the two regions wrote books about each others' civilization, history and other aspects which garnered a mutual interest between the two civilizations. A key result was that the Arab writers and poets wrote many books and eulogies about Pakistan, its problems, its cities and its famous personalities like Muhammad Ali Johar, Allama Muhammad Iqbal, and Muhammad Ali Jinnah and the like. This critical study not only surveys but also highlights the misconceptions found in literary works of Arab Scholars about historical facts concerned with political history of Muhammad Ali Jinnah.

Key Words:

Quaid Azam, Ali Jinnah, Arabic Literature

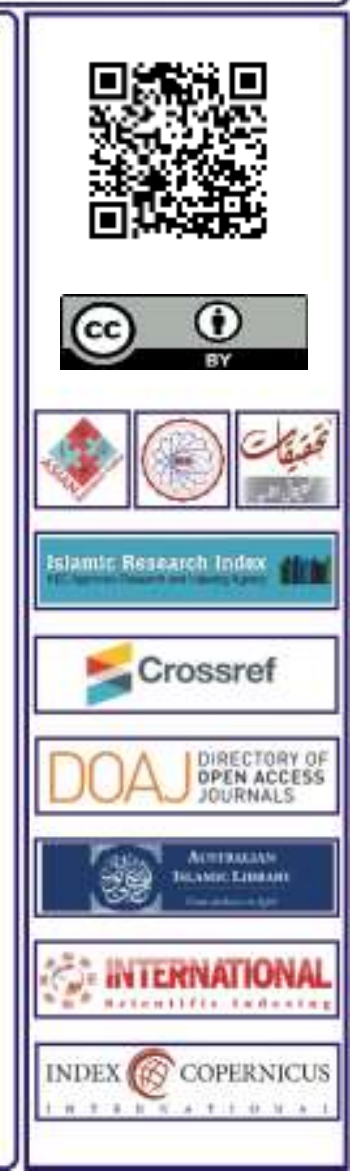


في العالم رجال كثيرون......ولكن الله ذكر نوعاً عزيزاً من الرجال تنويها بشأفم و تمجيداً لعلو مكانتهم فقال الله تعالى:

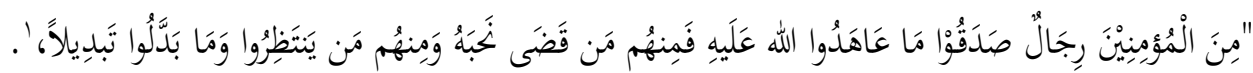

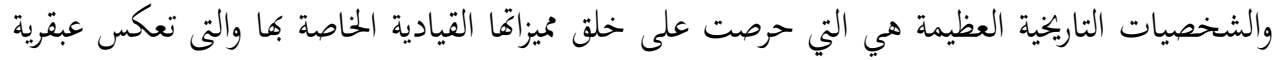

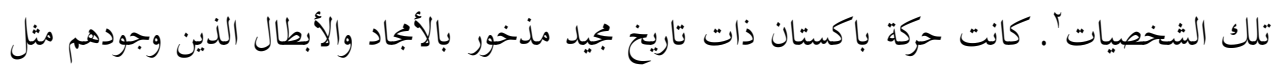

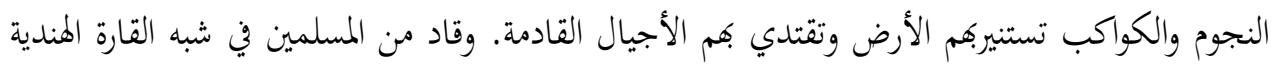

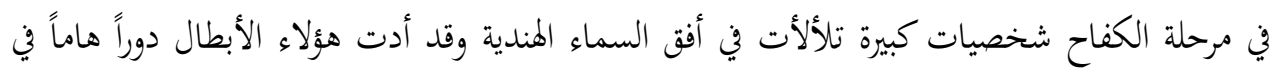

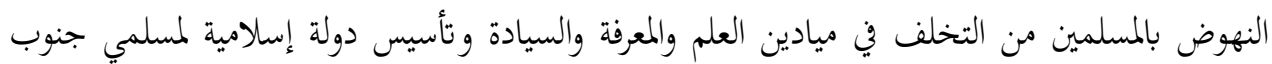
آسيا تمكنهم من تنظيم حياقم اليومية والاجتماعية والاقتصادية والسياسية على ضوء أحكام الشريعة

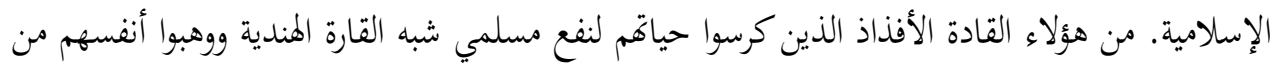

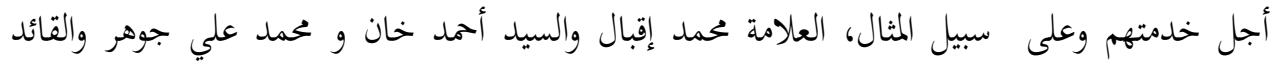

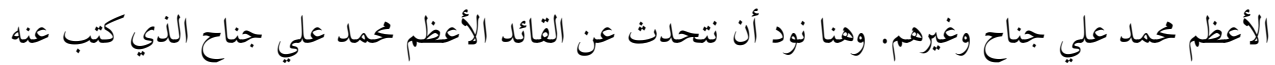
الأدباء والشعراء والكتاب العرب. لحمد علي جناح صفحات بمد في تاريخ حركة كفاح باكستان لأنه كان

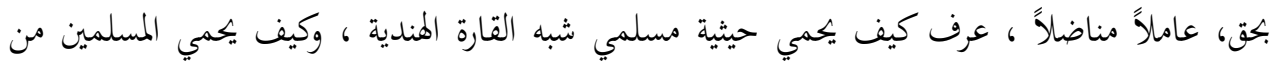
تورات الهنادكة والاستعمار الغربي. وموقفه الذي لم يتزعزع بين هذه الشدائد التي تزلزل موقفه بتعله بطلاً بحق

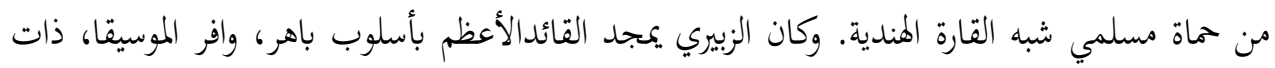

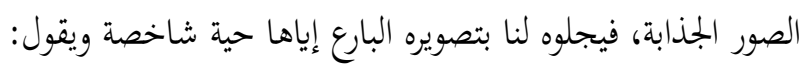

$$
\begin{aligned}
& \text { عيونٌ من الأقدارِ بتلوا لمغيبا }
\end{aligned}
$$

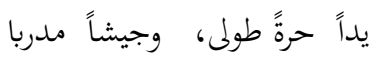

$$
\begin{aligned}
& \text { هبوب اليتامى ينشدون لمما أبا } \\
& \text { لها، استبانت أمرها المتشعبا لئابل } \\
& \text { وشكت، كمن قد شك فيها وكذبا } \\
& \text { وجيلاً من الإسلام ريان مخصبا }
\end{aligned}
$$

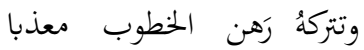

$$
\begin{aligned}
& \text { ويمنحهُ من ربقة الذِّلِ مُهربا } \\
& \text { يشق له منه طريقاً، ومذهباr }
\end{aligned}
$$
صحا من سناه الشعب والتف حوله

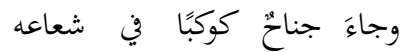
وهبت إليه...... أمةٌ من قبورها رأت نفسها في ضوئه، فكشفت إله وكانت، قدار ثابت قديماً بنفسها فألفت قوى مدخورةً في كياها وعزَّ عليها أن تراهُ مكبلاً فنادت جناحا كي يهلّ وثاقه ويعطيه وسط الديا جير مشعلاً 


\section{ميلاد القائد الأعظم محمد علي جناح:}

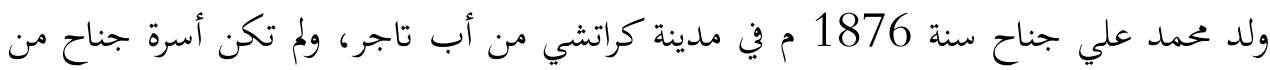
مدينة كراتشي أصلاً، بل كانت من مدينة كهتيوار؛ في ولاية كجرات التابعة لمدينة بمبئ. وكانت

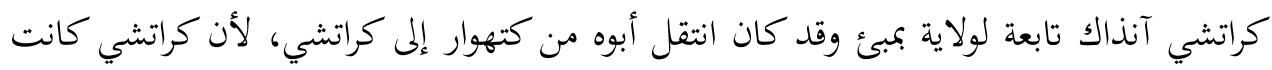

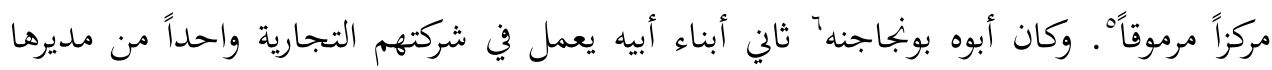

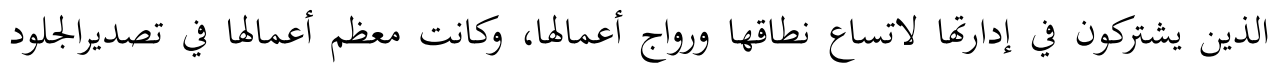

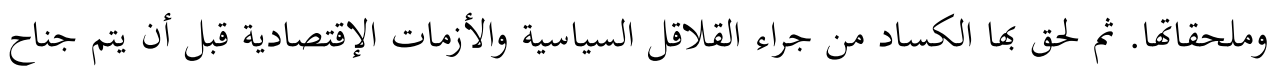
تعليمه في انجلترا حوالي سنة والأزمات الإقتصادية قبل أن يتم جناح تعليمه في النجلترا حوالي سنة

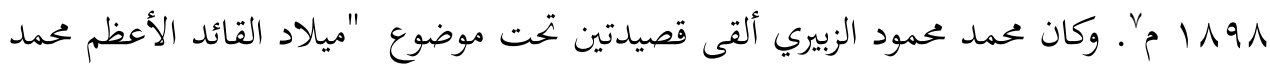
علي جناح" ومطلع أولمما:

عصورٌ من الديجور أنجبن كوكباً

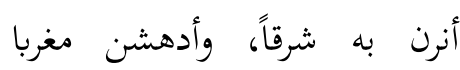

محون به أثامهُنَّ التّى مضت وهنت

وأهضن منه حظهُنَّ الذي كبا

وأخرجن شعباً، كان كنزاً مضيعاً

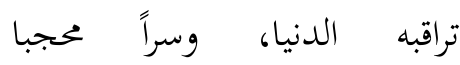

يعيش يسجن من رقئً، وطلاسم

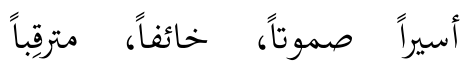

أخف من الأوهام وقعاً على النهى

وأنأى عن الموتى، وأخفى من الهبا

تطلَّ عليه الشمس عمياً مكيلةً

كأنَّ بها مساً من الشعر مغربا لُمديا

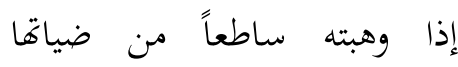

تحول من فعل الطلاسم غيهباء 


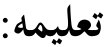

ولما نشأ وترعرع أرسله أبوه إلى إحدى مدارس بومبائي لتلقي تعليمه الإبتدائي و درس فيها فترة من الوقت، ولكن جو "بومبائي" لم يوافقه من جهة، ومن جهة أخرى لم تكن والثن والدته تستطيع فراقه طويلاً ولذا طلب مرة أخرى من بومبائي إلى كراتشي، و ألحق فيها بالمدرسة الثانوية "سندها منه

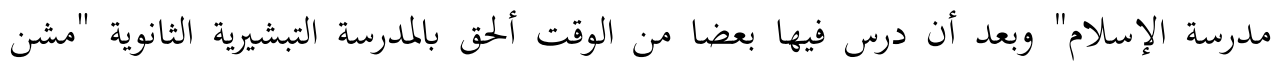
هائي سكول" ومن هذه المدرسة نجح في امتحان الشهادة الثانوية تحت إشراف "جامعة بومبائي"

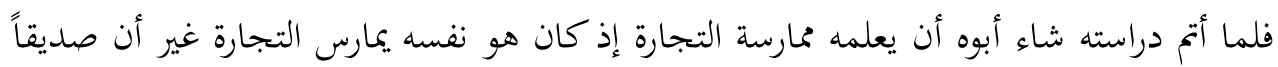

له قد أشار عليه بأن يبعث بإبنه إلى بريطانيا لمواصلة الدراسة العليا فيهاه أ. ذهب جناح إلى لندن وعاث فيها عيشة وسطاً كما يعيش كل الطلاب متوسطي الحال في بلاده

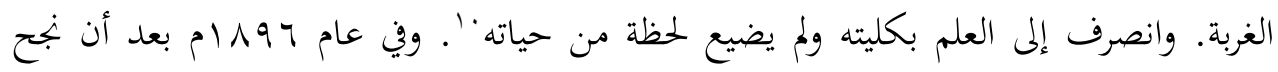

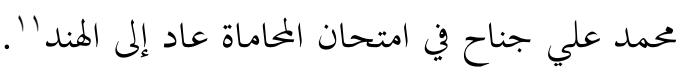
بدأ محمد علي جناح الشاب حياته كمحام بصورة مستقلة تماما؛ فلم يكن له أية خلفية ماعدا قدرته الفطرية و تصميمه وذكاء خارق كان يتمتع به وتمكن بفضل ذلك من بن التوصل إلى منزلة رفيعة بين المحامين في بومبائي، و حقق كل ذلك خلال سنوات قليلة وهذا إنجاز قلما حققه الآخرون من أمثاله.

\section{حياته السياسية:}

بعد أن فرغ محمد علي جناح من أموره الدنيوية الخاصة و دفع عن كاهل نفسه هم السعي إلى

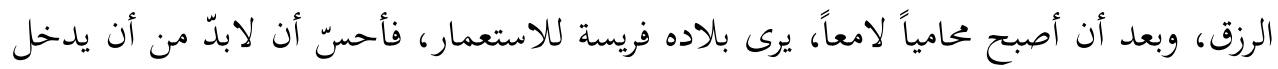

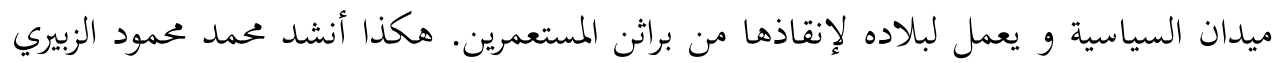

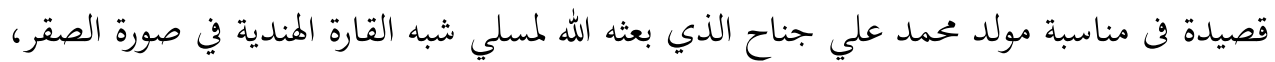
كما يصور الشاعر هذه الحقيقة فيقول:

عاتياً في هبوبه، وارتمائه

و تزجي به إلى عليائه

و استولدته في أجوائه

سراهُ تطايرت في هبائه

ملئ أرضه و وسمائه
ولد الصقر، و اثباً في فضائه

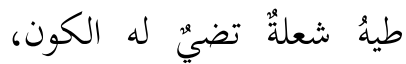
عاصفُ أسرعت بضعته الأقدار

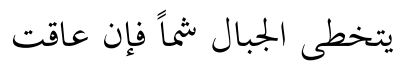
أطلقته يد الاله يمضي صاخباً 
ليس في قدرة السجون، ولا الأغلال تقييدُ عزمهِ،

$$
\text { و مضف مضئة كيف تبنى حيطان سجنٍ حوالى }
$$

سفير الوحدة:

كان محمد علي جناح عضواً لامعاً في البرلمان و خطيباً مفوها، وقانونياً بارعاً ولكنه لم يكن خطيباً

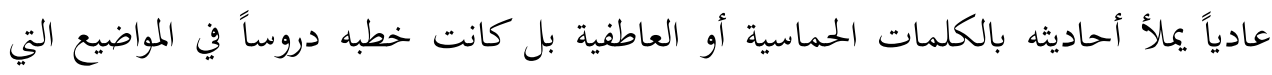
يعالجها. وكان قوله الفصل في كل ما يعالج من مواضيعء '.

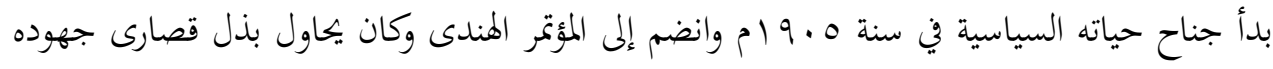
لوحدة المسلمين والهندوكيين. ومنذ سنة 7 ـ 9 ام يطلق عليه "سفيرالوحدة" أو سفير الوحدة الهندوكية الإسلامية؛ أ. أما عباس محمود العقاد فهو قدم أن في سنة الب ول ام يهتف البراهمة باسم رسول الوحدة. و د.أحمد معوض بين أن في 7 بو ام قامت السيدة نايدو بمحاولة للمصالحة بين الطائفتين (المسملون والهنادكة) فوصفها محمد علي جنه بأها نبية الوحدة الهند وإسلامية. قيام باكستان:

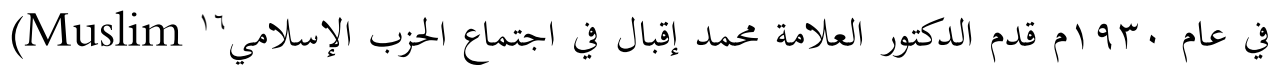
( League)

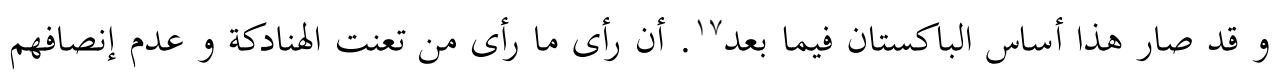

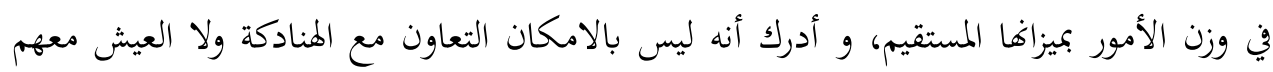

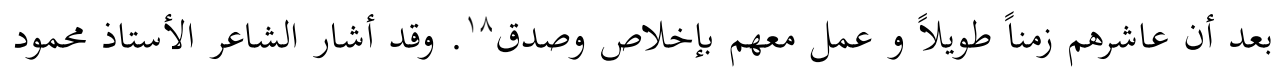
جبر '1 إلى هذه الحقيقة أن باكستان ثمرة عظيمة لمجهودات العلامة محمد إقبال فيقول:

بيت القصيد لشاعر موهوب

هل قبل ذلك مع حلم أديب؟

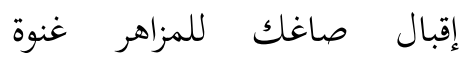

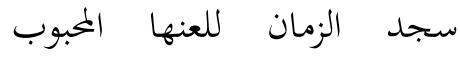
إقبال! باكستان أضحت روضة العهات 
معمورة بمغرد عشيب

صغت البيان قلائدا من عسجد

في رائق من رائع الأسلوب

ورأيت في الإسلام صدعا هائلا

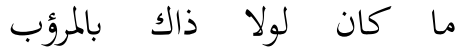

ماكان شعرا ما نظمت و و إنما

هو عبقر من فجك الملحوب

أرأيت من ورق تشاد مدائن؟

أم من سعير حديدها المصبوب.

أما الدكتور محمد كامل مرسي' فهو اعترف بصفات العلامة محمد إقبال الإستثنائية العالية يقول: "لم يكن إقبال ابن باكستان وحدها ولاخادم الشرق وحده ولا الحاني على العرب وحده بل كان من اولئك الآحاد الذين وهبوا أنفسهم لنفع بني آدم وخدمة الإنسانية"r"r. وقد أشار الشاعر السعودي محمد إبراهيم الغزاوي إلى جهوده و كفاحه لبلد مستقل لمسلمي شبه القارة الهندية ويقول:

على الذرى و بها الأجيال تلتزم

في (دولة) كشعاع الشمس ترتسم

بأن مرتعهم في بغيهم وخم

لو أننا بالأخاء الحق نلتئم

فهو (الوجود) ويخزي الكفر والعدم

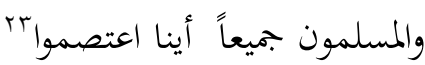

نظر أمامك (باكستان) شايخة

وقرعينا بأن الله أنشأها

وقل لمن شك تمن أنت تخطئهم

لنحن خير بني الانسان قد علموا

إذا اجتمعنا على (الايمان) من كثب

عاشت (باكستان) فيعز و رغد

وكانت نشاطات محمد إقبال في عام · ع ا |م حاسمة فبعد ما انتقد نظريات غاندي و سياساته و تصرفاته، وبعد الاقتناع باستحالة التفاهم مع حزب المؤتمر الهندوسي، ترأس في ب Y- Y آزار سنة • ع ام في لاهور اجتماعات الرابطة الإسلامية، وفي ذلك الاجتماع تم تبني قرار "حل باكستان" و اعتباره الهدف المنشور لمسلمي الهند، وطالب المسلمين بالاعتماد على الذات وقال: "انه لحلم رؤية المسلمين والهندوس وهم يعملون في سبيل هدف قومي موحد......ينتمي كل من المسلمين والهندوس إلى ديانتين مختلفين، و كل منهم فلسفاته وعاداته الإجتماعية و آدابه". 
وزاد جناح نشاطاته في عام اعـو (م)، وتركزت جميعها بابتاه إقامة باكستان، و آتت هذه الجهود

$$
\begin{aligned}
& \text { أكلها حيث باتت باكستان حقيقة مقبلة؛ ع........الخ } \\
& \text { منزلته عند العلامة محمد إقبال: }
\end{aligned}
$$

كان علامة محمد إقبال شجع محمد علي جناح لقيادة المسلمين وطلب منه أن يقود

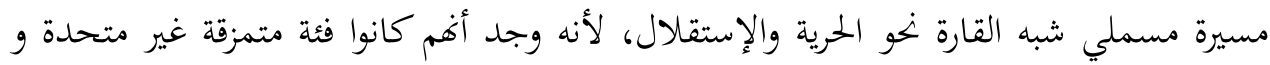

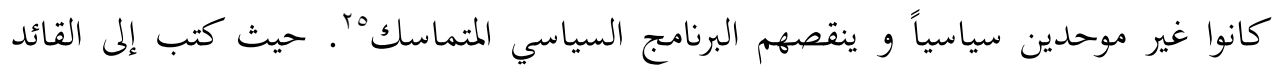

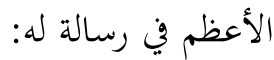

"إنك الشخص الوحيد في شبه القارة الهندية، الذي يحق له أن تعقد عليه الآمال في أنه سينقذنا

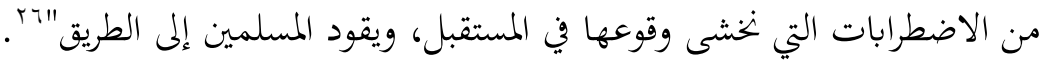
كفاحه وجهوده في قيام باكستان:

محمد على جناح هو أوّل رئيس دولة الباكستان ورأسها المفكر، فهو الذي أقام أسسها ودعم

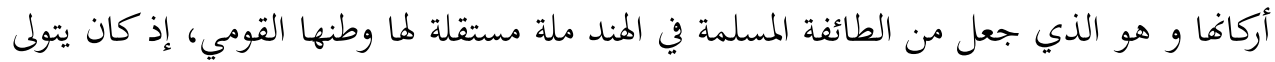
إرادة حركاما السياسية منذ سنين طويلة وينافح عن قضيتها بكل ما أوتى من قوة ولذا أطلق عليه بحق لقب "القائد الأعظم"rV. وقد أشار الشاعر محمد محمود الزبيري إلى أحوال مسلمي شبه القارة الهندية ودور محمد علي جناح ويقول:

$$
\begin{aligned}
& \text { فذابت في شعلة من ذكائه } \\
& \text { وتصدى جناح للمحنة الكبرى } \\
& \text { وطناً من جهاده، وبنائه } \\
& \text { أقبلت نهوه الملايين تبغي } \\
& \text { كل جرح لطِبه، ودوائه }
\end{aligned}
$$

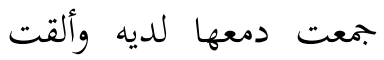

$$
\begin{aligned}
& \text { من روحه ومن إيحائه } \\
& \text { و أتته دنيا خرابٍ تريد البعث } \\
& \text { واستطارت نصره، و افتدائهيد } \\
& \text { فانبرى في نضاله يفتديها }
\end{aligned}
$$

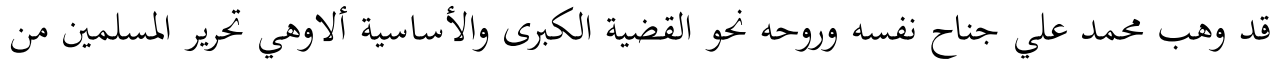
الهيمنة البريطانية و الهندوكية يحثهم على المطالبة بإقامة دولة مستقلة ذات سيادة في الأماكن التي

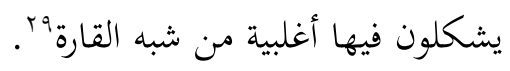


إمتاذ القائد الأعظم بميزات فذة ميزته عن سائر القادة والمفكرين وفي مقدمتها قدرقا القيادية

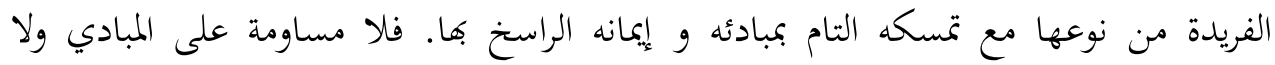
نكوص في الدعوة إلى تأسيس وطن قومي للمسلمين في شبه قارة جنوب آسيا.

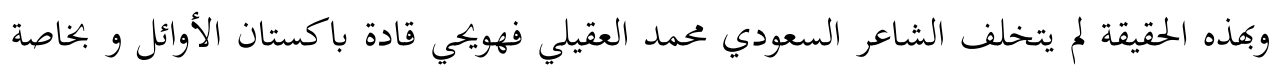

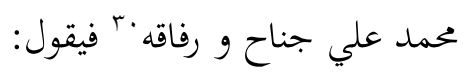

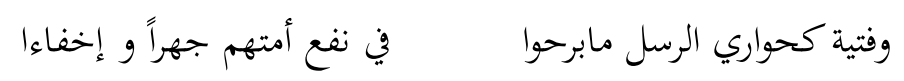

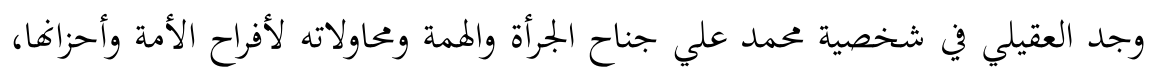
وهذه المساعي ذكرها الشاعر بقوله:

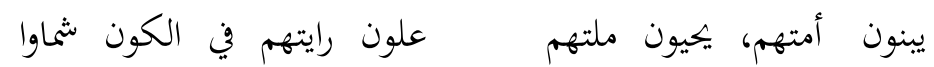

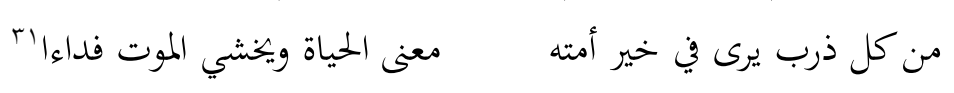

كان محمد علي جناح قائماً على موقف أن المسلين أمة منفصلة عن الأمة الهندوكية.

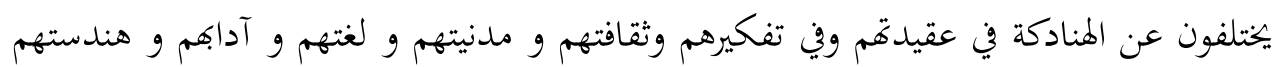

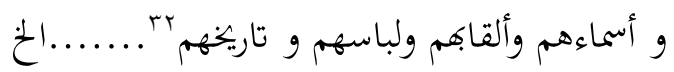

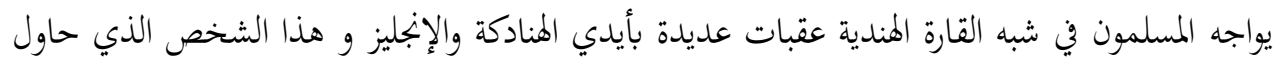
لنفعهم و دفعهم إلى كفاح للحصول على حريتهم. وقد يشير الشاعر عزيكته و أها لا تلين يقول:

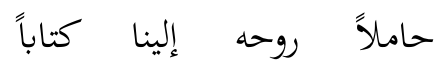
فيه ما في الماضى، ومن عظمائه يتلقيّ كلَّ امرئ مسلمٍ فيه دروس العليا عن آبائه يا لها من رسالةٍ في جناكِ

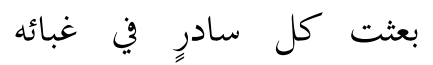

وقد يرجع الشاعر إلى أن مثال القائدالأعظم كمثال الأستاذ المربي الذي يعلم الارتفاع والشجاعة

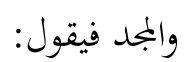
علمتنا كيف الخلاص من الضيم ومن قهره و من غلوائه وأريتنا كيف الطموح إلى المجد، وكيف الوثوب في أجوائه كل قلبٍ فيه جناح يريه فجه في سموه، وارتقائه يتلقىَّ دم الأسارى المواءَ الحرَّمن روحهه و من فئ أفيائه 
جعل الشاعر محمد علي جناح رمزاً للقوة وسيادة الاستبداد في مصارعة الهنود والمسلمين و قدمه في صورة البطل لأنه مأخوذ بالشجاعة والإقدام، يقول:

$$
\begin{aligned}
& \text { فيض أنفاسه يصوغ فضاءً }
\end{aligned}
$$

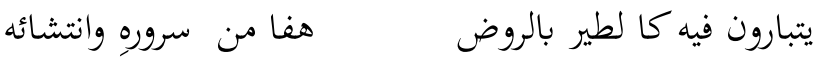

$$
\begin{aligned}
& \text { جاء المسلمون في الهند أسرى عصبياته و صرعى شقائه }
\end{aligned}
$$

وقد يشير الشاعر إلى دور الشعوب والقائد المدبر في طريق التحرر من القيود والاستعمار فيقول: عظم الخلف، واستبدَّ بأهليه، وضجوا من هوله، وبلائه وغد العيش مستحيلا، وضاف الشعب من أهله، ومن أبنائه

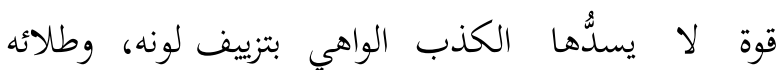

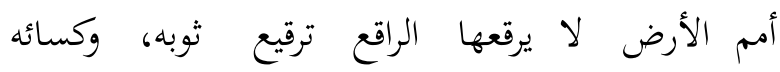
ومصير الشعوب كا لحقُ لاينيه بالٍ بوهم، وادعائه والملايين لاتعيش على الشك، ولا تستقر فوق هبائه هو خلف في الروح ماقيمة الرأي السياسي في لظى هوجائه مرجل الشعب لايبالي إذا جاش بأقطابه، ولا زعمائه

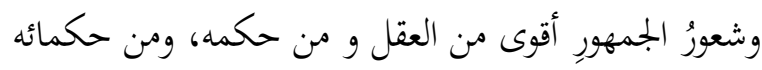

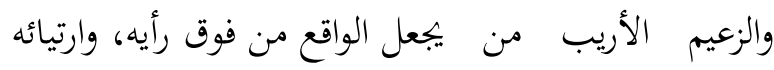
يتمشى مع النواميس لايعمل سير الورى على أهوائه بَّ.

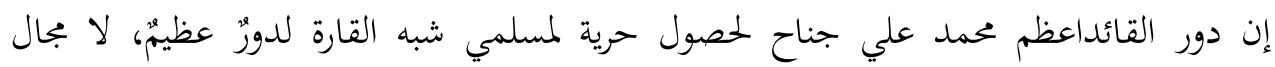
لإنكاره في الدراية والمهارة والتخطيط والتوجيه وبث الحماسة وبعث الحمية في مسلمي شبه القارة الهندية، و قد ذهب الشاعر إلى هذا فيقول: ومضت، في جهادها، كالأعاصير، تدوي بذكره، وندائه تلقى شذى السماءٍ، فتهتاجُ، هياج الحريق في هو جائه تهرئ و تذيب القيود، في لهب الروح، وتمحو الشقاءَ عن أشقيائه وإذا بالسجون توي، وبالسجان يبغي الفكاك من سجنائه

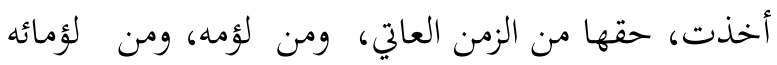


وفي آخر القصيدة يؤيد الشاعر أن مجد الحياة مقصود بسيادة الإسلام و لامجد للمسلم إلا بسيادة

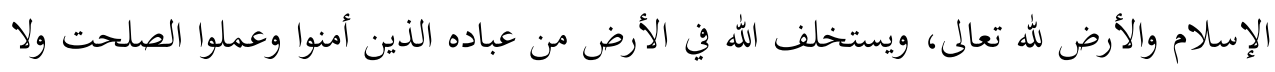

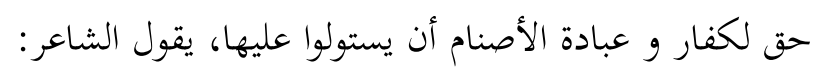

$$
\begin{aligned}
& \text { قد تولى الغازي، ومات فموتوا }
\end{aligned}
$$

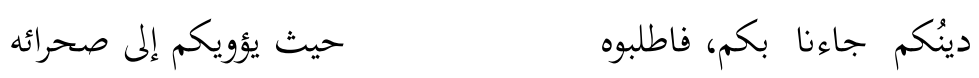

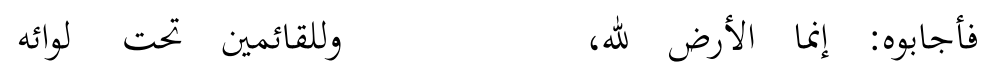

$$
\begin{aligned}
& \text { هي ملكُ لمن دحاها، وليست مِلكَ أنداده،ولا شركائه } \\
& \text { ولمن ييذلون أرواحهم فيه نشاوى بحبه وليه ولائه } \\
& \text { ولمن جابهو المصارع، والأهوال، شوقا لوصله ولقائه } \\
& \text { نحن أنصاره، أحق بدنياه، و وأولى بأرضِهِ، وسمائه } \\
& \text { نحن أقدارة نشيدُ كما شادَ، وتبني أكفنا كبنائه } \\
& \text { نخن أطواده، بجوف الثرى نرسو، وتعلوها ماتنا في فضائه }
\end{aligned}
$$

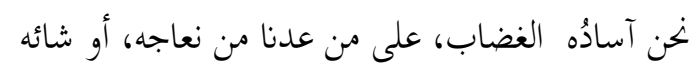

$$
\begin{aligned}
& \text { نخن حراس اسمه، الواحد القهار في ملكه، وفي كبريائه }
\end{aligned}
$$

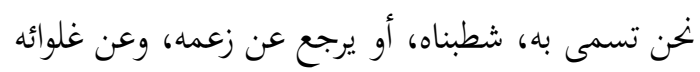

$$
\begin{aligned}
& \text { سوف نبني ملكاً طهوراً، و نسترخص فيه أرواحنا لفدائه }
\end{aligned}
$$

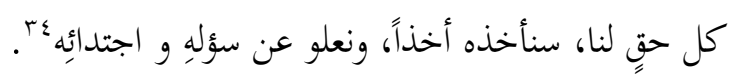


اهتمامه بالقضايا الإسلامية:

لم ينتظر الزعيم محمد علي جناح قيام دولة باكستان طويلاً حتى يتدخل في شؤون المسلين و

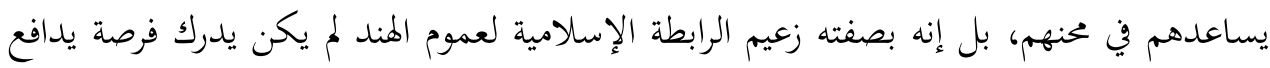
هما عن المسلمين إلا اهتبلها، فقد كانت له موقف في الدفاع عن أفغانستان و إيران و العراق

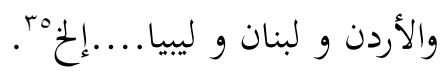

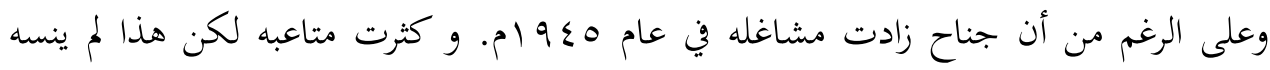
فلسطين ففي هب مارس كتب إلى السيد أتلي رئيس و زراء البريطانيا يطلب منه أن يوضع سياسة

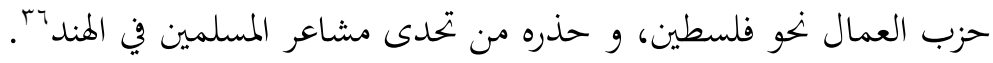
وكان قد تلقى رسالة شكر من الإمام يحي ملك اليمن على موقف باكستان من القضية الفلسطينية و تأييدها للصالح العربي فكتب إليه يقول: "أشارككَ يا صاحب الجلالة تماما في دهشتك و غضبك نهو انعدام العدالة الذي أبدته منظمة الأمم المتحدة، بقرارها غير العادل حول فلسطين، و أوكد كذلك ثانية لإخوة العرب بأن باكستان ستقف إلى جانبهم و ستبذل كل ما يمكن لتأييدهم و مساعدةم في معارضتهم لقرار الأمم

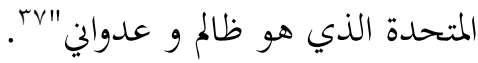
شغل شعراء العالم العربي و الإسلامي في العصر الحاضر بمكانة باكستان في العالم الإسلامي

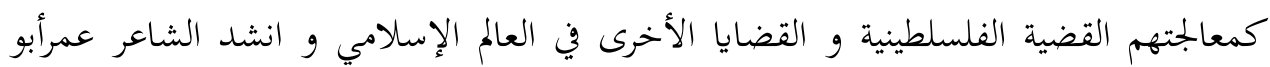
قوص ^־صيدة جميلة بعنوان "باكستان حامية الإسلام و منقذة المسلين" يقول في مطلعها:

$$
\begin{aligned}
& \text { في ليلة قمراء ذات بهاء } \\
& \text { أرسلت روحي في الفضاء طليقة لترى شؤن الناس من عليهاء }
\end{aligned}
$$

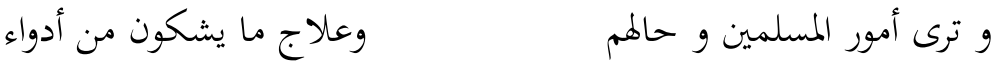

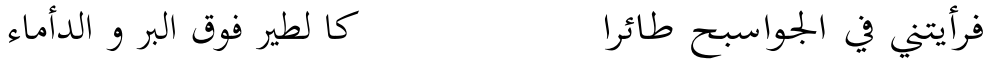

$$
\begin{aligned}
& \text { ورأيت أنوار المدائن كلها }
\end{aligned}
$$

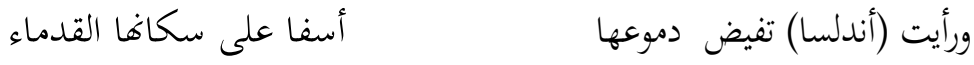

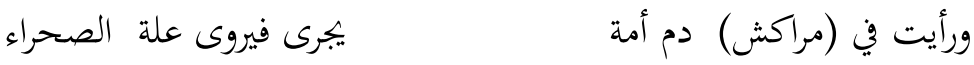

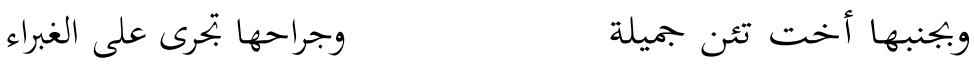

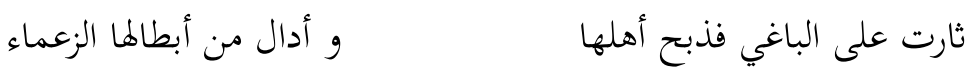




$$
\text { فنسألت أين المسلمون ليذكروا }
$$

ويظل يصاعد من نبرة الأمل ينتهي إلى البيت الأخير كاشفاً عن مكانة باكستان و دوره الإصلاح الأمة المسلمة يشمله بتحقيق هذا الأمل، مبدداً ما جثم من ظلمات. و نجد أن الشعراء قد تنبهوا إلى مكانة باكستان و غرض قيامها التي يشع نورها على الكون و يستنشق غيرها العالم الإسالامي في مشرقه و مغربه فأحالوا عليها في شعرهم و استخدموها رمزاً للجهاد والنضال والنبل يقول:

$$
\text { فأجاب (باكستان) صوت لم يزل }
$$

رثاء حممد على جناح:

تركت قرائح الشعراء والأدباء بموت البطل المؤسس محمد علي جناح في 11 ستمبر سنة مـ 9 |م أم بعد تأسيس الدولة بعام كامل والمصائب والعقبات التى وقعت ضد المسلمين أثناء التقسيم وهجرة الهندوس إلى مناطق أكثريتهم في الهند، وترك المسلمون دورهم ومتاعهم في الهند وهاجروا إلى باكستان ليقيموا في مخيمات و يبدأوا حياةً جديدة. تثير هذه الظروف أحاسيس الأدباء و الشعراء، و قد رثا عدد من الشعراء العربية القائد الباكستاني محمد علي جناح. منهم على سبيل المثال الدكتور صوفي محمد ضياء الحق المتوفي عام 9 هو ام الذي أنشد قصيدة طويلة و مطلعها:

$$
\begin{aligned}
& \text { بعقوتنا غراب البين صباحا أصاب الموت ائدنا }
\end{aligned}
$$

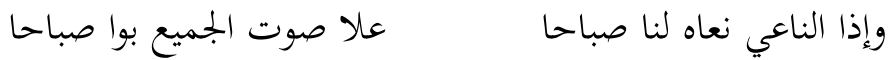

$$
\begin{aligned}
& \text { رمى الحدثان قوم المسلمينا لم يحدوا من القدر البراحا }
\end{aligned}
$$


إن شخصية محمد علي جناح- رحمه الله- تميزت بعلمها و قوتما كجبل شامخ لايسلم من ارتطام الأمواج في قاعه وسفحه و بخاصة في مثل موقعه في تأسيس دولة مستقلة للطائفة المسلمة في المند

$$
\text { و لذلك فقد قدم الشاعر وصفه في الأبيات التالية يقول: }
$$

$$
\text { وأخوثقة وذو رأى سديد }
$$

نظمت قصائد و قطعات في حياة محمد علي جناح و بعد موته و استمرت إلى العصر الحديث. والشعراء ولأدباء يعرضون هذه الشخصية لناشئة الأمة و يذكرون مجده و عظمته. وعند ما جاء

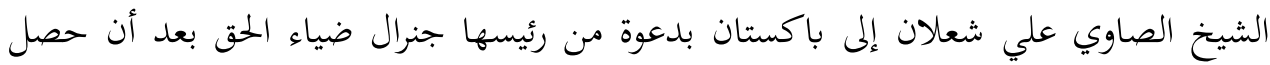
على ميد الية ذهبية من سماحته بمناسبة ترجماته لشعر إقبال، و ان هذا في عام 971 ام، زار الشيخ ضريح القائد الأعظم في كراتشي و أنشد هناك قصيدة طويلة تناول فيها بطولات القائد و

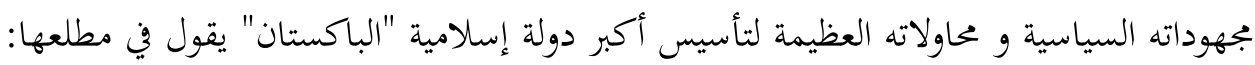

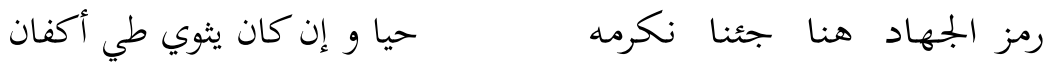

$$
\begin{aligned}
& \text { أكاد أسمع من خلف الستور صدى يخاطب الشعب من جنات ورضوان }
\end{aligned}
$$

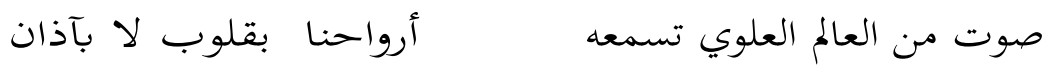

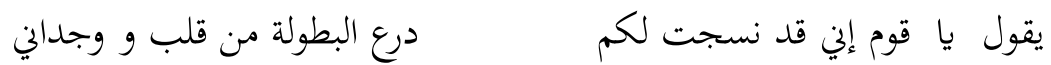

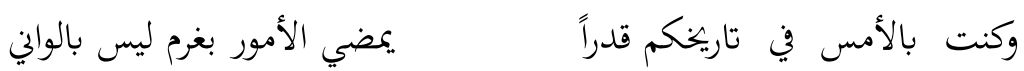

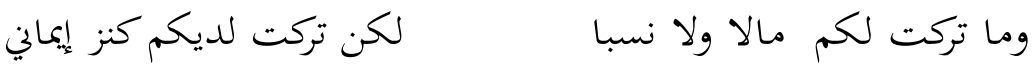

$$
\begin{aligned}
& \text { عزمي و حزمي و تصميمي و مقدرتي وهمتي و مؤاساتي و عرفاني }
\end{aligned}
$$

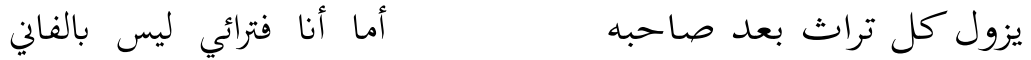

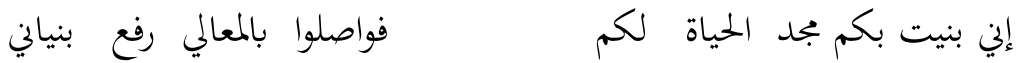

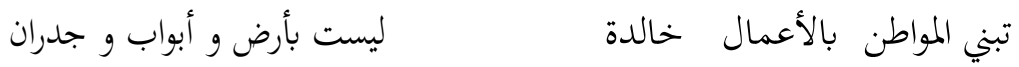

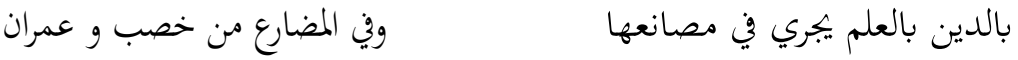

$$
\begin{aligned}
& \text { ذكراي يوم حساب عن تقدمكم على تواصل أجيال و أزمان }
\end{aligned}
$$

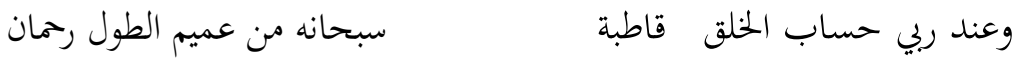

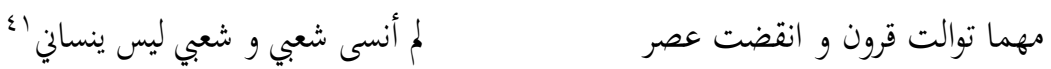




\section{(c) This work is licensed under a Creative Commons Attribution 4.0 International Licence.}

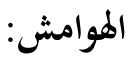

1 Al-Ahzab: 23

2 Mujjala Pakistan al-Mussawera, al-dairatul aama lil-Aflam wal Matbooat wazara tul alaam wa tatweer alsahafa, alhakoomat albakistania, islamamabad, November, December, 2001, safa 5

3 Muhammad Mahmood Alzubaire, dewan alzubaire, taqdeem, abdul aziz maqaleh, darul odat berot, 360-361/2

Aslan hazhe alkalma "kahtayawad" wa hea min walayat alhind.

5 D. Ehsan haqi, Muhammad ali jinah bani bakistan hayatuhu wa sayasatuhu, taba/1, darul fkar dimashq, sorya, 1987, safa18

6 Aslan hazhe alkalma fi urdia, boonja jinah.

7 Abbas mahmood aluqad, alaamal alkamla, (tarajum wa sayar6), darul kutab allabnani bairot, qad akhta almualaf fe biyan alsana1898, aslan rajah Muhammad ali jinah fi alsana 1897.

8 Muhammad mahmood alzubaire, dewan alzubaire, 360/1, 2.

9 Muhammad Hassan alazmii, zainab haim alhakeem bilishtrak, janat alarz Kashmir, tabba/1, dar ehya alkutab alarbia 1949, P 181-182. Wa alustaz Fazal mahbod azeem, Alqaid alazam Muhammad ali jinah, majalla aldarasat alislamia, V 11, adad/4, majma albahoos alislamia, Islamabad, zolhajja 1397hijri, december1976, P/40.

10 D.Ehsan haqi, Muhammad ali jinah, p 19.

11 Majala aldarasat alislamia, juz11, v 4, P 22.

12 Al ustaz Sharif almujahid, Alqaid alazam Muhammad ali jinah hayathu wa enjazathu, majala bakistan almusawara, juz2, adad9, muderia alaflam walmatboat, wazarat alaalam wal ezaat, hakoma albakistania, Islamabad, P 4.

13 Dewan Alzubaire, juz1, 2, safa 373-374.

14 D.Ehsan haqi, Muhammad ali jinah, P 22

15 Albaijoom shaista sahrwardi Ikramullah, nehzat alfikar alislami wa mulid bakistan" Awahae, V3, qism alsahafa safara bakistan, bairoot Lubnan, Aghastas 1967, P10, wa D Ali Anwar, qadat albakistan, bakistan almusawira, V2, adad10, muderia alaflaam walmatboat hakomat bakistan, Islamabad, P25.

16 Yastamilu alarab ahyanan kalma alhizb alislami wa ahyanan hum yastamilon alrabita alislamia.

17 Majjala aldarasat alislamia, V11, adad4, P26

18 D.Ehsan haqi, Muhammad ali jinah, P26-28.

19 Lam ajid tarjumathu

20 Iqbal fe sher alarbi, jamma o tahqeeq wa tarjama, D Zahoor Ahmad Azhar, almaktaba alilmia lahor, tabba1, 1977, P109.

21 Lam ajid tarjumathu

22 Muhammad kamil mursi, Iqbal min olaaika alahad, Iqbal alarab ala dirasat Iqbal(jamma taqdeem:d zahoor Ahmad azhar, P22.

23 Alwaee, v 33, aghustus 1960, P 13

24 Al Qaid alazam Muhammad ali jinah, taareeb:D sohail zikar, (taqdeem)P 20 
25 Alustaz sharif almujahid, alqaid al azam hayatuhu wa injazatuhu, majala bakistan almusawira, V2, adad 9, P8.

26 Aldarasat alislamia, V 11, adad 4, P5

27 Muhammad Hassan al aazme bil ishtirak zainab alhakeem, janat alarz Kashmir, P 181

28 Muhammad mahmood alzubaire, dewanuhu nuqta fi zulam, darul odat bairot, 1986, V 2, P149.

29 Majalla bakistan almusawira, vofember December 2001, P8.

30 Yaani alzuama alajala alazeen sahamo fe tasees bakistan Muhammad ali Jinnah mislu Liyaqar ali khan.

31 D Abdullah alhamid, alsher alhadis fe mumlikatul Arabia alsaudia, tabba2, darulkutab alsaudi alriyaz, 1993, p 185

32 D ehsan haqi, Muhammad Ali jinah, p 151

33 Dewan alzubaire, V2, p 146-147

34 Nafsul marjih, p 149-150

35 D ehsan haqi, Muhammad Ali jinah, p 216

36 Al Qaid alazam Muhammad ali jinah, taareeb:D sohail zikar, P 22.

37 Nafsul marjih, p 23.

38 Lam ajid tarjumathu

39 Alwaee, V43, 42 mayu 1960, qism alsahafa sifarah bakistan bairot labnan, p48

40 D.Ahmad Idrees, Aladab alarabi fi shib alqara alhindia.majalla holiah aljamiha alislamia, v5, aljamiha alislamia, Islamabad, 1997, p161.

41 Aiwan e Iqbal, Iqbal Acadmy bakistan lahor, p255 